\title{
WILEY-VCH
}

DOI: 10.1002/ ((please add manuscript number))

Article type: Full Paper

\section{Inducing elasticity through oligo-siloxane crosslinks for intrinsically stretchable semiconducting polymers}

Ging-Ji Nathan Wang, Leo Shaw, Jie Xu, Tadanori Kurosawa, Bob C. Schroeder, Jin Young Oh, Stephanie J. Benight and Zhenan Bao*

G. N. Wang, L. Shaw, Dr. J. Xu, Dr. T. Kurosawa, Dr. B. C. Schroeder, Dr. J. Y. Oh, Dr. S. J. Benight, Prof. Z. Bao

Department of Chemical Engineering, Stanford University, Stanford, CA 94305-5025, USA

E-mail: zbao@stanford.edu

Keywords: stretchable, semiconducting polymers, elastic, OFET

The promise of wearable and implantable devices has made stretchable organic semiconductors highly desirable. Though there are increasing attempts to design intrinsically stretchable conjugated polymers, their performance in terms of charge carrier mobility and maximum fracture strain are still lacking behind extrinsic approaches (i.e. buckling, Kiragarmi interconnects). Here we apply polymer crosslinking with flexible oligomers as a strategy to reduce the tensile modulus and improve fracture strain, as well as fatigue resistance for a high mobility diketopyrrolopyrrole (DPP) polymer. These polymers are crosslinked with siloxane oligomers to give stretchable films stable up to a strain $\varepsilon=150 \%$ and 500 strain/release cycles of $100 \%$ strain without the formation of nano-cracks. Organic field-effect transistors are prepared to assess the electrical properties of the crosslinked film under cyclic strain loading. An initial average mobility $\left(\mu^{a v g}\right)$ of $0.66 \mathrm{~cm}^{2} / \mathrm{Vs}$ is measured at $0 \%$ strain. A steady $\mu^{a v g}$ above $0.40 \mathrm{~cm}^{2} / \mathrm{Vs}$ is obtained in the direction perpendicular to the strain direction after 500 strain/release cycles of $20 \%$ strain. The $\mu^{a v g}$ in the direction parallel to strain, however, is compromised due to the formation of wrinkles.

\section{Introduction}

The advancement of conjugated polymers in the past decade has been remarkable. From pursuing amorphous silicon to surpassing its benchmark charge carrier mobility $(\mu)$ of 1.0 $\mathrm{cm}^{2} / \mathrm{Vs}$, today's conjugated polymers have obtained hole mobilities well above $10 \mathrm{~cm}^{2} / \mathrm{Vs},{ }^{[1-4]}$ 


\section{WILEY-VCH}

approaching the performance of polycrystalline silicon. While there continues to be interest in further improving these polymers' electrical performance, the next challenge lies in realizing their full potential in stretchable electronics for wearable and implantable devices such as displays,${ }^{[5,6]}$ sensors,${ }^{[7,8]}$ and artificial skin. ${ }^{[9-12]}$

Common extrinsic approaches to stretchability include mechanically designed metallic serpentine or Kiragarmi interconnects ${ }^{[13-16]}$ and induced "buckling." ${ }^{[6,17-19]}$ Though both are excellent methods to impart stretchability on rigid materials, their elaborate fabrication have limited their potential in low-cost, printable electronics. ${ }^{[20]}$ The development of intrinsically stretchable polymer semiconductors, on the other hand, has just recently begun. The challenges in developing such materials arise from the conflicting material design rules for good electronic properties versus robust mechanical properties. The ideal polymer morphology for electrical transport is one that is at least semicrystalline, while the ideal morphology for mechanical compliance is amorphous with low glass transition temperature. ${ }^{[21,22]}$ Recent approaches to intrinsically stretchable semiconductors include side-chain engineering, backbone segmentation, and physical blending with amorphous polymers. ${ }^{[23-27]}$ To date, the best performing stretchable semiconductor is regioregular poly(3-hexylthiophene)-co-polyethylene di-block copolymer, which is stable up to $600 \%$ strain as measured from a $50 \mu \mathrm{m}$ drop-cast film. However, its electrical property was compromised, with a $\mu^{\max }$ of $0.02 \mathrm{~cm}^{2} / \mathrm{Vs}$ at $0 \%$ strain, and its mobility at higher strains was not measured. ${ }^{[28]}$

The reported approaches essentially apply the same strategy of increasing the solid film amorphous fractions and lowering the tensile modulus, which softens the materials and allow them to be stretched to greater strains without forming cracks. "Softness" however is insufficient for stretchable electronics. So far, all of the works referenced have, at most, focused on the electrical performance of soft conjugated polymers under an applied stress, but their behavior upon relaxation or under multiple cycles of loading is seldom discussed. ${ }^{[29]}$ For practical applications of stretchable electronics, it is required that once stress is released, these 


\section{WILEY-VCH}

polymer films must exhibit the same properties as they did prior to stretching. While softer materials may be the answer to fracture strain enhancement, a stretchable material needs to exhibit resistance to fatigue and provide robust electrical performance after repeated strain/release cycles. Therefore, elasticity is an essential property for intrinsically stretchable conjugated polymers, as it allows the material to retain structural integrity.

The crosslinking of polymer networks is a well-known strategy for realizing elastomers because it prevents irreversible sliding between polymer chains. Even though it typically leads to an increase in elastic modulus, studies have shown that crosslinking in conjugated polymers can suppress crystallization by reducing polymer chain rearrangement and aggregation. ${ }^{[30,31]}$ Furthermore, if a soft, flexible crosslinker is used, crystallinity may be reduced. Previously, the crosslinking of conjugated polymers was widely utilized in polymer light-emitting diodes (PLED) to (1) reduce aggregation and stabilize emission and (2) to form an insoluble layer for multi-layer device fabrication. ${ }^{[30-33]}$ However, its effect on the mechanical and transport properties of conjugated polymers was not investigated. The polymer films were typically crosslinked by introducing crosslinkable polymer end-capping groups such as styrene ${ }^{[31,32]}$ and acrylates. ${ }^{[33]}$ Polymer-chain-end crosslinking is non-ideal for stretchable electronics, as a low molecular weight (MW) polymer is often required to achieve a relatively high crosslinking density. On the other hand, a high MW is typically needed for good charge transport. ${ }^{[2,34]}$ Moreover, chain-end crosslinking may disrupt charge transport along the backbone.

In this work we present the synthesis and characterization of a 3,6-di-2-thienylpyrrolo[3,4-c]pyrrole-1,4-dione-(DPP)-based conjugated polymer crosslinked with a poly(dimethylsiloxane) (PDMS) oligomer through the former's side-chains. Siloxanes are wellknown flexible polymers with $\mathrm{Si}-\mathrm{O}-\mathrm{Si}$ bond angles that can vary between $135^{\circ}-180^{\circ}$, whereas DPP polymers are among the best performing, high-mobility conjugated polymers. ${ }^{[2,3]}$ Sidechain crosslinking is ideal because high crosslinking density could be easily tuned and disruption of charge transport is minimized (Figure 1b). Our covalently crosslinked network 


\section{WILEY-VCH}

demonstrates an increase in elasticity, with no nano-crack formation observed after 500 strain/release cycles at strains of $\varepsilon=100 \%$ and 1000 strain/release cycles of $\varepsilon=20 \%$. Organic field-effect transistors (OFET) of the crosslinked films exhibit a $\mu^{a v g}$ of $0.66 \mathrm{~cm}^{2} / \mathrm{Vs}$ at $0 \%$ strain and maintained their initial mobility upon relaxation from $100 \%$ strain. The strained crosslinked films maintained a $\mu^{a v g}>0.4 \mathrm{~cm}^{2} / \mathrm{Vs}$ after 500 strain/release cycles of $\varepsilon=20 \%$. To our knowledge, this is the first study on the effect of crosslinking on both the mechanical and the electric properties of conjugated semiconducting polymers.

\section{Results and Discussion}

\subsection{Materials design and Synthesis}

We synthesized a series of DPP-based random co-polymers with varying densities of alkene-terminated linear side-chains for crosslinking with H-terminated PDMS (Figure 1a). The successful incorporation of crosslinkable side-chains was confirmed by NMR (Figure S2). The polymers were end-capped (EC) with 0.06 eq. of 2-bromo-thiophene during polymerization to control their number average molecular weight $\left(M_{\mathrm{n}}\right)$ for better processability. ${ }^{[35]}$ Hightemperature size exclusion chromatography (HT-SEC) of the polymers showed comparable $M_{\mathrm{n}}$ $\sim 20 \mathrm{kDa}$ (Figure 1c). The large weight dispersity $\left(\bigoplus_{\mathrm{M}}\right)$ indicates that the polymers have a strong tendency to aggregate even at $180{ }^{\circ} \mathrm{C}$. The higher the percentage of linear side-chains, the greater the $\bigoplus_{M}$ and degree of aggregation ${ }^{[36]}$ (Figure S17). OFETs of the polymers all exhibited high hole mobilities above $1.0 \mathrm{~cm}^{2} / \mathrm{Vs}$ and on/off ratios above $10^{5}$. Previously, mobility as high as $10.5 \mathrm{~cm}^{2} / \mathrm{Vs}$ was reported for this class of polymer for $M_{\mathrm{n}}$ of $110 \mathrm{kDa} \cdot{ }^{[2]}$ In our case, the mobility is reduced due to the incorporation of poorly soluble linear side-chains, which led to a non-optimal molecular weight. A linear, H-terminated PDMS oligomer with $\sim 10$ repeating units was selected as our soft crosslinker for a higher degree of flexibility and reactivity. ${ }^{[37]}$

The semiconductors were crosslinked by hydrosilylation, which is an efficient and heavily utilized reaction for the preparation of PDMS elastomers. Crosslinking must be carried out post-deposition, as crosslinking renders the polymer insoluble and impossible to process. 


\section{WILEY-VCH}

The PDMS oligomer was spincoated together with the catalyst and conjugated polymer, followed by vacuum annealing at $80{ }^{\circ} \mathrm{C}$ for 30 minutes to form the final crosslinked film. The crosslinking of the films was monitored by attenuated total-reflectance Fourier transform infrared spectroscopy (ATR-FTIR). As shown in Figure 2a, the Si-H stretching and bending peaks near 2,100 $\mathrm{cm}^{-1}$ and $900 \mathrm{~cm}^{-1}$ respectively as well as the two vinyl bending peaks near $950 \mathrm{~cm}^{-1}$ were clearly visible prior to crosslinking. However, after crosslinking, the $\mathrm{Si}-\mathrm{H}$ peaks gradually disappeared, and the emergence of a new peak could be seen around $1,130 \mathrm{~cm}^{-1}$ from the new Si-C bond formed. The intensity of the two vinyl peaks decreased but did not completely disappear as a 1:0.6 vinyl/Si-H ratio was used to reduce the amount of unreacted siloxane and improve crosslinking efficiency.

\subsection{Mechanical properties}

The dichroic ratio, $R=A / / / A_{\perp}$, where the peak absorbance $(A)$ of the film with polarized light parallel $(/ /)$ to the strain direction is divided by that of the perpendicular $(\perp)$, was measured to investigate the polymer alignment under strain. ${ }^{[38]}$ This is a useful method to assess the formation of micro-cracks, as the dichroic ratio increase will slow down when stress is dissipated by crack-propagation as supposed to chain alignment. ${ }^{[39]}$ As shown in Figure 2c, crosslinked polymers 10DPPTTECx and 20DPPTTECx show a steady linear increase in $R$ up to $150 \%$ strain, whereas their non-crosslinked counterparts 10DPPTTEC and 20DPPTTEC as well as our control polymer - branched-DPPTTEC, show a decrease in slope after $100 \%$ strain. The crack formation at 100\% strain was further confirmed by optical microscopy (Figure $\mathbf{2 b}$ ). Large micro-cracks could be seen for 20DPPTTEC, whereas 20DPPTTECx showed no sign of cracks even at $\varepsilon=150 \%$. A strain greater than $150 \%$ was not applied due to the rupture of the PDMS substrate.

The tensile modulus of branched-DPPTTEC, 20DPPTTEC, and 20DPPTTECx was measured by mechanical buckling metrology ${ }^{[21,23,40]}$ and provided in Figure 2e. We observed a slight increase in elastic modulus for 20DPPTTEC after incorporating linear side-chains, 


\section{WILEY-VCH}

possibly due to an increase in aggregation ${ }^{[36]}$, consistent with decreased polymer solubility and higher $\bigoplus_{M}$ measured by HT-SEC. The elastic modulus of 20DPPTTECx, on the other hand, is 200 MPa lower despite being a crosslinked film, thus accounting for its improved ductility.

The yield strength as determined by the buckling onset strain ${ }^{[41]}$ of the polymers was also improved upon crosslinking. Following a similar procedure in literature, polymer films were transferred onto Ecoflex 50, stretched, and relaxed in increments of $1 \%$ strain for each stretch/relax cycle until wrinkles could be observed under the optical microscope (Figure S8). A low-modulus substrate was selected for easy and clear wrinkle formation for a more precise determination of the yielding point. 20DPPTTEC began to yield and show wrinkles at the film edge at $8 \%$ strain, whereas the crosslinked film only started to yield at $14 \%$ strain, indicating the crosslinked film is more elastic.

\subsection{Film morphology}

To analyze the microstructure and compare the crystallinity of the thin films, X-ray diffraction was performed at grazing incidence for 20DPPTTEC and 20DPPTTECx (Figure 3). The intensities were scaled with exposure time, X-ray path length, and film thickness so that the color scales are normalized and linear, allowing for comparison across each diffraction image. While the Q values of intensity exactly on the meridian are approximate because the detector is planar, the images were not adjusted to remove those regions in order to make visual comparison easier. In all of the images, strong out-of-plane peaks from the $(h 00)$ reflections can be seen up to third order, indicating crystalline and ordered lamella with a $Q(100)$ value of $0.32 \AA^{-1}$ corresponding to a d-spacing of $19.6 \AA$. Adjacent to this peak at a Q value of $1.27 \AA^{-}$ ${ }^{1}$ is a strong out-of-plane reflection (indicated with a yellow arrow). It is likely that some of this scattering is due to alkyl side-chain packing that often produces a diffuse halo in this region of $\mathrm{Q}$, in addition to weak fourth-order lamella intensity. There is an in-plane peak around $1.47 \AA^{-}$ ${ }^{1}$ corresponding to the crystalline OTMS layer of the functionalized substrate. 


\section{WILEY-VCH}

The $\pi$-stacking (010) peak centered around $1.74 \AA^{-1}$ (or $3.61 \AA$ in real space) is observed in all cases and indicates that polymer crystallites are primarily oriented edge-on; the weak outof-plane halos at $\mathrm{Q}=1.72 \AA$ and $1.94 \AA$ are aberrant shadows from the beamstop. As expected, when comparing the overall diffraction intensities of the images, the thermally annealed samples are consistently more crystalline than those as-cast. The crosslinked films also have decreased crystallinity compared to the un-crosslinked, which is apparent in the meridian linecut plots of the polymer films (Figure S15). This is in agreement with our hypothesis that polymer crystallinity could be decreased by introducing flexible crosslinkers, which increases the difficulty for polymer molecules to rearrange and pack. Furthermore, the decrease in crystallinity accounts for the decrease in elastic modulus of the crosslinked films.

The surface morphology information of the polymer films was obtained by tapping mode AFM. As shown in Figure 4, the AFM height image of 20DPPTTEC annealed at $160{ }^{\circ} \mathrm{C}$ under vacuum has an interconnected nanofibrillar morphology similar to that reported for branched-DPPTTEC. ${ }^{[42]}$ For the crosslinked film 20DPPTTECx, a uniform polymer fiber morphology was maintained but a decrease in fibrillary crystal domain size is evident (figure 4). This supports the GIXD result of decreased crystallinity upon crosslinking and is in accordance with the decrease in hole mobility for their respective OFET.

\subsection{Electrical Characterizations}

OFETs of the crosslinked films exhibited decrease in the average mobility $\left(\mu^{a v g}\right)$ due to the introduction of insulating siloxanes and the decrease in film crystallinity. As shown in Figure 2d, the greater the amount of siloxane added, the lower its mobility. Nonetheless, 10DPPTECx and 20DPPTTECx still have moderate $\mu^{a v g}$ of $0.90 \mathrm{~cm}^{2} / \mathrm{Vs}$ and $0.66 \mathrm{~cm}^{2} / \mathrm{Vs}$ respectively, whereas 40DPPTTECx has a $\mu^{\text {avg }}$ of $0.38 \mathrm{~cm}^{2} / \mathrm{Vs}$. All polymers exhibit on-off ratios $>10^{5}$ (Table S1).

20DPPTTECx was selected for further device and mechanical testing because of its good charge transport properties and efficient crosslinking. The crosslinking density of 


\section{WILEY-VCH}

10DPPTECx was too low to be effective, and the films were often still slightly soluble when dipped into chloroform (Figure S9). On the other hand, 20DPPTTECx was more reliable, the thin films soaked in chloroform were consistently insoluble and could still form working OFETs (Figure S10 and Table S2).

We also investigated the effect of strain on the device performance of the stretched films. Conventional methods for evaluating the electrical properties of strained films typically involve back-transfer of the polymer film from its PDMS host to an $\mathrm{OTMS}-\mathrm{SiO}_{2}$ substrate. The transfer, however, does not work well for our crosslinked film as the siloxane crosslinkers could covalently bind to the PDMS substrate by (1) silanol exchange reaction and (2) hydrosilylation of unreacted alkenes and silanes in the PDMS. Hence we utilized a soft-contact lamination method, ${ }^{[25,39]}$ which allowed us to measure the mobilities of our polymer films directly on the PDMS substrate (Figure 5a). This is a more convenient method as it does not require multiple transfers of the semiconducting active layer and the deposition of drain and source electrodes. However, the mobilities obtained are generally lower compared with mobilities from traditional bottom-gate, top-contact (BGTC) devices with films directly deposited, as shown in Figure 5c. This is because soft-contact lamination is sensitive to the surface roughness of the polymer film since it could prevent intimate contact between the dielectric and the semiconducting layer. BGTC devices have a smoother dielectric-semiconductor interface as the polymer films are directly spincoated onto the dielectric surface from solution. This interfacial contact issue becomes more severe later when the film is subjected to large strains and repeated strain/release cycles, as wrinkles are formed under these conditions.

The mobilities of 20DPPTTEC and 20DPPTTECx films were measured at various strain levels for the cases when the transistor channel length is oriented parallel $(/ /)$ and perpendicular ( $\perp$ ) to the strain direction. We observed a decrease in $\mu / /$ and $\mu \perp$ for both polymers at $100 \%$ strain (Figure 6a, b). This was expected for the pristine polymer as micro-cracks could be observed by optical microscopy; but not the crosslinked films since no cracks could be observed. During 


\section{WILEY-VCH}

measurements, we noticed wrinkles temporarily forming upon strain due to the Poisson effect, under which the film experiences a compressive strain in the direction perpendicular to strain (Figure S11). As soft-contact lamination is highly sensitive to the surface roughness of the active layer, the films were relaxed back to $\varepsilon=0 \%$ from their respective strains, and their transport properties were re-measured. Interestingly, upon releasing strain, the $\mu \perp$ fully recovered for 20DPPTTECx whereas 20DPPTTEC only partially recovered. 20DPPTTECx maintained its starting $\mu_{\perp} \sim 0.4 \mathrm{~cm}^{2} / \mathrm{Vs}$ up to $100 \%$ strain. On the other hand, we observed a further decrease in $\mu / /$ from both polymers upon relaxation.

AFM was used to investigate the film morphology of the relaxed films, which may account for the decrease in $\mu / /$, as well as to look for nano-cracks that may not have been detected by optical microscopy. Figure 7a shows wide cracks on 20DPPTTEC after relaxation from $100 \%$ strain. On the contrary, wrinkles as tall as $20 \mathrm{~nm}$ were found on the surface of 20DPPTTECx, but no cracks were visible. In fact, no cracks could be observed on the crosslinked film even when stretched to $100 \%$ strain for 500 cycles (Figure 7b).

We suspect the wrinkles account for the decrease in $\mu / /$ of both films upon relaxation. Wrinkles are formed by polymer delamination and yielding during strain, followed by relaxation of the PDMS host, which supplies a compressive force on the deformed film. The wrinkles lead to poor contact between the dielectric and semiconductor, hence a reduction in charge transport. However, these wrinkles - contrary to the ones formed from Poisson effect are perpendicular to strain direction and thus have less impact on $\mu_{\perp}$. Instead, these wrinkles greatly obstruct the charge transport pathway in the direction parallel to strain. This explains why $\mu \perp$ could recover upon film relaxation, whereas we observe a further decrease in $\mu /$. This is in contrast to the literature, which typically reports a boost in $\mu_{/ /}$and a compromise in $\mu_{\perp}$ upon strain attributed to chain alignment. ${ }^{[38]}$ 


\section{WILEY-VCH}

To assess the fatigue resistance of our crosslinked films, OFET performance of 20DPPTTEC and 20DPPTTECx were further investigated under cyclic loading at 20\% strain. This strain was purposely selected to be well below the fracture limit of both polymers but above the range for most stretchable applications. The AFM height profile of each sample was measured after their respective loading cycles. From the AFM images, we see wrinkles forming in both films after 10 strain/release cycles at 20\% strain (Figure S13), which accounts for the relatively lower $\mu / /$ compared to $\mu_{\perp}$. 20DPPTTECx gave stable $\mu \perp$ up to 500 strain/release cycles at 20\% strain (Figure 8a) and with no nano-cracks detected by AFM up to 1,000 strain/release cycles, whereas 20DPPTTEC started to form 5-nm-deep nano-cracks (Figure S14) after 10 cycles, accompanied by a sharp decrease in mobility.

The root-mean-squared (RMS) roughness of the films were calculated from the AFM images and plotted against loading cycles. As shown in Figure 8a, 20DPPTTEC had a sharper increase in RMS roughness with increasing strain/release cycles than the crosslinked polymer. Its RMS roughness started to plateau after 10 cycles due to the formation of nano-cracks, which allowed stress to dissipate. On the other hand, 20DPPTTECx had siloxane chains to "lock" the conjugated chains in position, reducing irreversible deformation from stretching and hence exhibiting an improvement in elasticity and resistance to fatigue with a slower increase in RMS roughness. The AFM images of the crosslinked films after their respective strain/release cycles show smoother films and fewer wrinkles formed compared with its non-crosslinked counterpart (Figure S13).

Previous work has shown that an increase in dielectric RMS roughness can lead to an exponential decrease in mobility. ${ }^{[43]}$ Likewise, we expect a similar trend for our system if the compromise in mobility of our crosslinked film is mainly due to an increase in roughness of our active layer caused by wrinkle formation. Indeed, when plotting our 20DPPTTECx mobilities $\mu$ against RMS roughness, we observed an exponential decay in $\mu$ with increasing RMS roughness (Figure 8b), thus suggesting an inverse correlation between $\mu$ and wrinkle 


\section{WILEY-VCH}

formation. Interestingly for 20DPPTTEC, two separate regimes could be observed. Similar to 20DPPTTECx, we initially observed a "wrinkle-dominant" regime, where the exponential decay in $\mu$ is due to wrinkle formation. But after 10 strain/release cycles at $20 \%$ strain, we observed a "crack-dominant" regime marked by a sharp increase in decay rate. This sharp decay was attributed to the formation of nano-cracks, which had a smaller effect on the RMS roughness of the film than the wrinkles formed from earlier cycles but a detrimental effect on charge carrier mobility. Figure $8 \mathrm{~b}$ further supports our hypothesis that the mobility decay in the crosslinked film was mainly due to wrinkle formation instead of crack propagation.

\section{Conclusion}

This paper explores crosslinking as a strategy for improving mechanical robustness of intrinsically stretchable semiconducting polymers. We successfully developed such strategy for a high mobility DPP polymer using linear PDMS oligomers attached by hydrosilylation and confirmed by ATR-FTIR. The crosslinked films exhibited an increase in elasticity followed by improvement in film ductility and fatigue resistance. Our 20DPPTTECx was stretchable up to $150 \%$ strain without crack formation, as verified by optical microscope, AFM, and optical dichroic ratio measurements and gave a stable $\mu \perp$ up to $100 \%$ strain. The OFETs of 20DPPTTEC and 20DPPTTECx were characterized in detail under cyclic loading at $20 \%$ strain. The crosslinked film showed a stable $\mu \perp \sim 0.4 \mathrm{~cm}^{2} / \mathrm{Vs}$ up to 500 strain/release cycles of $20 \%$ strain by soft-contact lamination, whereas a sharp decrease in $\mu_{\perp}$ was observed after 10 strain/release cycles for 20DPPTTEC. AFM images showed that 20DPPTTECx had a greater resistance towards wrinkle and crack formation from cyclic loading and, hence, stable electric performance.

Previous approaches for stretchable polymer semiconductors have mainly focused on developing softer, low-modulus materials, but "softness" does not address the problem of plastic deformation under cyclic loading. Ultimately, there is a need to develop materials that are elastic while maintaining good electrical performance. While there has been work on 


\section{WILEY-VCH}

crosslinking conjugated polymers within the PLED community, this is the first study on its effect on the polymer semiconductor film's mechanical as well as charge transport properties. When appropriate crosslinkers are chosen, crosslinking can both increase elasticity and soften conjugated polymers. Lastly, our work brings attention to wrinkling as a potential device failure mechanism for stretchable semiconductors. While wrinkles due to yielding is less detrimental than crack propagation, it is a different failure mode that needs to be addressed if we hope to develop practical stretchable devices. Proper selection of substrates and other layers of the device component will be necessary.

\section{Experimental Section}

OFET Device Fabrication and Characterization: OFET devices were fabricated on highly doped n-type $\mathrm{Si}(100)$ wafer with a 300 -nm-thick thermal $\mathrm{SiO}_{2}$, which was modified with an octadecyltrimethoxysilane (OTMS) self-assembled monolayer according to our reported method ${ }^{[44]}$ The OTMS-treated substrate was rinsed with toluene, acetone and isopropyl alcohol, and then dried with nitrogen before use. The organic semiconductor thin films were spun-cast on the $\mathrm{SiO}_{2} / \mathrm{Si}$ substrates at a rate of $1200 \mathrm{rpm}$ for $60 \mathrm{~s}$ from polymer solutions in chloroform ( $5 \mathrm{mg} / \mathrm{mL}$ ). The films were vacuum-annealed at $160{ }^{\circ} \mathrm{C}$ for $30 \mathrm{~min}$. For BGTC devices, topcontact gold electrodes $(40 \mathrm{~nm})$ were subsequently deposited by thermal evaporation through a shadow mask with the channel length $(L)$ and width $(W)$ defined as 50 and $1000 \mu \mathrm{m}$ respectively.

For soft-contact lamination OFETs, the annealed polymer films were transferred to a 0.5 mm-thick PDMS (Dow Corning Sylgard 184, crosslinker to prepolymer ratio of 1:10 (1/1)) and directly laminated on an OTMS-treated 300-nm $\mathrm{SiO}_{2} / \mathrm{Si}$ substrate with gold source and drain electrodes $(L=50 \mu \mathrm{m}, W=1000 \mu \mathrm{m})$. All measurements of the transistors were conducted using a Keithley 4200 semiconductor parameter analyzer (Keithley Instruments Inc, Cleveland, $\mathrm{OH}, \mathrm{USA}$ ) under ambient atmosphere at room temperature.

Preparation of crosslinked films: To a $4 \mathrm{~mL}$ vial was added nDPPTTEC (5 mg) and anhydrous chloroform $(1 \mathrm{~mL})$. The polymer solution was vigorously stirred at $80{ }^{\circ} \mathrm{C}$ for $2 \mathrm{~h}$ and then 


\section{WILEY-VCH}

hydride terminated polydimethylsiloxane $(\mathrm{cSt}=2-5 ; \sim 726 \mathrm{~g} / \mathrm{mol})(0.23,0.47$ and $0.95 \mu \mathrm{L}$ for $\mathrm{n}$

$=10,20$ and 40 respectively) and Karstedt's catalyst (platinum(0)-1,3-divinyl-1,1,3,3tetramethyldisoloxane complex solution in xylene, $\mathrm{Pt} \sim 2 \%)(0.2 \mu \mathrm{L})$ were added. After stirring the solution for an additional $1.5 \mathrm{~h}$, the crosslinked polymer films were spin-cast on OTMS modified $\mathrm{SiO}_{2} / \mathrm{Si}$ substrates at a spin rate of $1200 \mathrm{rpm}$ for $60 \mathrm{~s}$. The films were vacuum-annealed at $80{ }^{\circ} \mathrm{C}$ for $30 \mathrm{~min}$ to complete crosslinking, followed by $160{ }^{\circ} \mathrm{C}$ for device fabrication.

\section{Supporting Information}

Supporting Information is available from the Wiley Online Library or from the author.

\section{Acknowledgements}

This work was supported by Kuraray Company and the Air Force Office of Scientific Research (FA9550-15-1-0106). G.N.W. acknowledges the Croucher Foundation for financial support. L. S. acknowledges support from the Kodak Graduate Fellowship. J.X. and J.Y.O. acknowledges Samsung Electronics. T. K. acknowledges support from the Office of Naval Research (N0001414-1-0142). B.C.S. acknowledges the National Research Fund of Luxembourg for financial support (project 6932623). Use of the Stanford Synchrotron Radiation Lightsource, SLAC National Accelerator Laboratory, is supported by the U.S. Department of Energy, Office of Science, Office of Basic Energy Sciences under Contract No. DE-AC02-76SF00515.

Received: ((will be filled in by the editorial staff))

Revised: ((will be filled in by the editorial staff)) Published online: ((will be filled in by the editorial staff))

[1] H. R. Tseng, L. Ying, B. B. Y. Hsu, L. a. Perez, C. J. Takacs, G. C. Bazan, A. J. Heeger, Nano Lett. 2012, 12, 6353.

[2] J. Li, Y. Zhao, H. S. Tan, Y. Guo, C.-A. Di, G. Yu, Y. Liu, M. Lin, S. H. Lim, Y. Zhou, H. Su, B. S. Ong, Sci. Rep. 2012, 2, 754.

[3] I. Kang, H.-J. Yun, D. S. Chung, S.-K. Kwon, Y.-H. Kim, Kang, H.-J. Yun, D. S. Chung, S.-K. Kwon, Y.-H. Kim, J. Am. Chem. Soc. 2013, 135, 14896.

[4] B. Nketia-Yawson, H.-S. Lee, D. Seo, Y. Yoon, W.-T. Park, K. Kwak, H. J. Son, B. Kim, Y.-Y. Noh, Adv. Mater. 2015, 27, 3045.

[5] J. Liang, L. Li, X. Niu, Z. Yu, Q. Pei, Nat. Photonics 2013, 7, 817. 


\section{WILEY-VCH}

[6] M. S. White, M. Kaltenbrunner, E. D. Głowacki, K. Gutnichenko, G. Kettlgruber, I.

Graz, S. Aazou, C. Ulbricht, D. A. M. Egbe, M. C. Miron, Z. Major, M. C. Scharber, T.

Sekitani, T. Someya, S. Bauer, N. S. Sariciftci, Nat. Photonics 2013, 7, 811.

[7] X. Huang, Y. Liu, H. Cheng, W.-J. Shin, J. A. Fan, Z. Liu, C.-J. Lu, G.-W. Kong, K.

Chen, D. Patnaik, S.-H. Lee, S. Hage-Ali, Y. Huang, J. A. Rogers, Adv. Funct. Mater.

2014, 24, 3846.

[8] H.-H. Chou, A. Nguyen, A. Chortos, J. W. F. To, C. Lu, J. Mei, T. Kurosawa, W.-G.

Bae, J. B.-H. Tok, Z. Bao, Nat. Commun. 2015, 6, 8011.

[9] S. J. Benight, C. Wang, J. B. H. Tok, Z. Bao, Prog. Polym. Sci. 2013, 38, 1961.

[10] M. L. Hammock, A. Chortos, B. C. K. Tee, J. B. H. Tok, Z. Bao, Adv. Mater. 2013, 25, 5997.

[11] R. C. Webb, A. P. Bonifas, A. Behnaz, Y. Zhang, K. J. Yu, H. Cheng, M. Shi, Z. Bian, Z. Liu, Y.-S. Kim, W.-H. Yeo, J. S. Park, J. Song, Y. Li, Y. Huang, A. M. Gorbach, J.

A. Rogers, Nat. Mater. 2013, 12, 938.

[12] D. J. Lipomi, M. Vosgueritchian, B. C.-K. Tee, S. L. Hellstrom, J. A. Lee, C. H. Fox, Z. Bao, Nat. Nanotechnol. 2011, 6, 788.

[13] J. A. Rogers, T. Someya, Y. Huang, Science. 2010, 327, 1603.

[14] Y. Zhang, H. Fu, Y. Su, S. Xu, H. Cheng, J. A. Fan, K.-C. Hwang, J. A. Rogers, Y. Huang, Acta Mater. 2013, 61, 7816.

[15] Z. Song, X. Wang, C. Lv, Y. An, M. Liang, T. Ma, D. He, Y.-J. Zheng, S.-Q. Huang, H. Yu, H. Jiang, Sci. Rep. 2015, 5, 10988.

[16] D.-H. Kim, J. Xiao, J. Song, Y. Huang, J. A. Rogers, Adv. Mater. 2010, 22, 2108.

[17] D. J. Lipomi, B. C.-K. Tee, M. Vosgueritchian, Z. Bao, Adv. Mater. 2011, 23, 1771.

[18] M. Kaltenbrunner, M. S. White, E. D. Głowacki, T. Sekitani, T. Someya, N. S. Sariciftci, S. Bauer, Nat. Commun. 2012, 3, 770.

[19] Y. Lee, M. Shin, K. Thiyagarajan, U. Jeong, Macromolecules 2016, 49, 443. 


\section{WILEY-VCH}

[20] S. R. Forrest, Nature 2004, 428, 911.

[21] B. O’Connor, E. P. Chan, C. Chan, B. R. Conrad, L. J. Richter, R. J. Kline, M. Heeney, I. McCulloch, C. L. Soles, D. M. DeLongchamp, ACS Nano 2010, 4, 7538.

[22] R. Noriega, J. Rivnay, K. Vandewal, F. P. V. Koch, N. Stingelin, P. Smith, M. F. Toney, A. Salleo, Nat. Mater. 2013, 12, 1038.

[23] S. Savagatrup, A. D. Printz, D. Rodriquez, D. J. Lipomi, Macromolecules 2014, 47, 1981.

[24] A. D. Printz, S. Savagatrup, D. J. Burke, T. N. Purdy, D. J. Lipomi, RSC Adv. 2014, 4, 13635.

[25] M. Shin, J. Y. Oh, K.-E. Byun, Y.-J. Lee, B. Kim, H.-K. Baik, J.-J. Park, U. Jeong, Adv. Mater. 2015, 27, 1255.

[26] R. Peng, B. Pang, D. Hu, M. Chen, G. Zhang, J. Mater. Chem. C 2015, 3, 3599.

[27] E. Song, B. Kang, H. H. Choi, D. H. Sin, H. Lee, W. H. Lee, K. Cho, Adv. Electron. Mater. 2016, 2, n/a.

[28] C. Müller, S. Goffri, D. W. Breiby, J. W. Andreasen, H. D. Chanzy, R. A. J. Janssen, M. M. Nielsen, C. P. Radano, H. Sirringhaus, P. Smith, N. Stingelin-Stutzmann, Adv. Funct. Mater. 2007, 17, 2674.

[29] D. J. Lipomi, Adv. Mater. 2016, 28, 4180.

[30] W. Zhao, T. Cao, J. M. White, Adv. Funct. Mater. 2004, 14, 783.

[31] A. R. Davis, J. a. Maegerlein, K. R. Carter, D. Marsitzky, J. Murray, J. C. Scott, K. R. Carter, E. Scheler, I. Bauer, P. Strohrigl, W. Zhao, T. Cao, J. M. White, J. Am. Chem. Soc. 2011, 133, 20546.

[32] D. Marsitzky, J. Murray, J. C. Scott, K. R. Carter, Chem. Mater. 2001, 13, 4285.

[33] E. Scheler, I. Bauer, P. Strohrigl, Macromol. Symp. 2007, 254, 203.

[34] H. N. Tsao, D. M. Cho, I. Park, M. R. Hansen, A. Mavrinskiy, D. Y. Yoon, R. Graf, W. Pisula, H. W. Spiess, K. Müllen, J. Am. Chem. Soc. 2011, 133, 2605. 


\section{WILEY-VCH}

[35] M. J. Robb, D. Montarnal, N. D. Eisenmenger, S. Ku, M. L. Chabinyc, C. J. Hawker, Macromolecules 2013, 46, 6431.

[36] T. Lei, Y.-C. Lai, G. Hong, H. Wang, P. Hayoz, R. T. Weitz, C. Chen, H. Dai, Z. Bao, Small 2915, 11, 2946.

[37] A. Nyczyk, C. Paluszkiewicz, M. Hasik, M. Cypryk, P. Pospiech, Vib. Spectrosc. 2012, $59,1$.

[38] B. O’Connor, R. J. Kline, B. R. Conrad, L. J. Richter, D. Gundlach, M. F. Toney, D. M. DeLongchamp, Adv. Funct. Mater. 2011, 21, 3697.

[39] H.-C. Wu, S. J. Benight, A. Chortos, W.-Y. Lee, J. Mei, J. W. F. To, C. Lu, M. He, J. B.-H. Tok, W.-C. Chen, Z. Bao, Chem. Mater. 2014, 26, 4544.

[40] C. M. Stafford, C. Harrison, K. L. Beers, A. Karim, E. J. Amis, M. R. VanLandingham, H.-C. Kim, W. Volksen, R. D. Miller, E. E. Simonyi, Nat. Mater. 2004, 3, 545.

[41] A. D. Printz, A. V Zaretski, S. Savagatrup, A. S. Chiang, D. J. Lipomi, ACS Appl. Mater. Interfaces 2015, 7, 23257.

[42] Y. Li, S. P. Singh, P. Sonar, Adv. Mater. 2010, 22, 4862.

[43] M. L. Chabinyc, R. Lujan, F. Endicott, M. F. Toney, I. McCulloch, M. Heeney, Appl. Phys. Lett. 2007, 90, 233508.

[44] Y. Ito, A. A. Virkar, S. Mannsfeld, J. H. Oh, M. Toney, J. Locklin, Z. Bao, J. Am. Chem. Soc. 2009, 131, 9396. 
WILEY-VCH

a

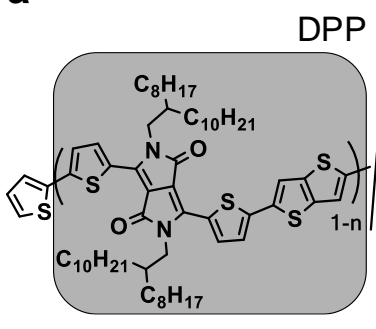

DPP polymer

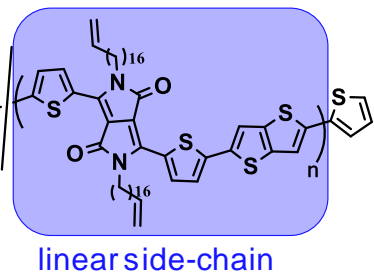

$\begin{aligned} \text { branchedDPPTTEC } & =0 \% \\ \text { 10DPPTTEC } & =10 \% \\ \text { 20DPPTTEC } & =20 \% \\ \text { 40DPPTTEC } & =40 \%\end{aligned}$

Siloxane Crosslinker

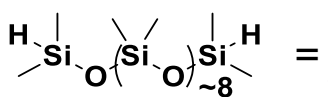

$\mathrm{H}$-terminated PDMS $\sim 726 \mathrm{~g} / \mathrm{mol}$

C

\begin{tabular}{lccc} 
& $M_{n}(\mathrm{kDa})$ & $M_{w}(\mathrm{kDa})$ & $\bigoplus_{M}$ \\
\hline branchedDPPTTEC & 27.0 & 100 & 3.71 \\
10DPPTTEC & 20.4 & 85.2 & 4.18 \\
20DPPTTEC & 16.5 & 66.0 & 4.00 \\
40DPPTTEC & 15.6 & 75.0 & 4.80
\end{tabular}

b

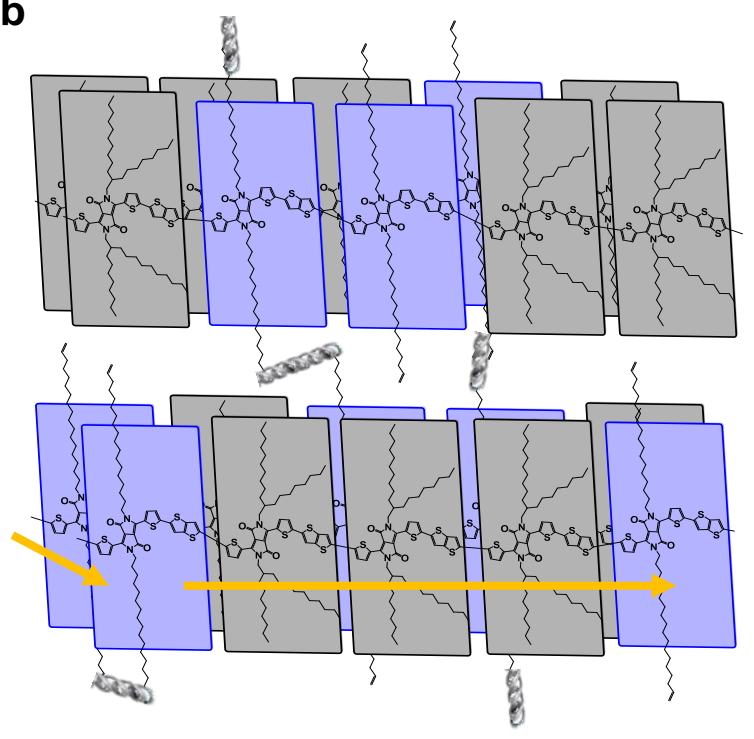

d

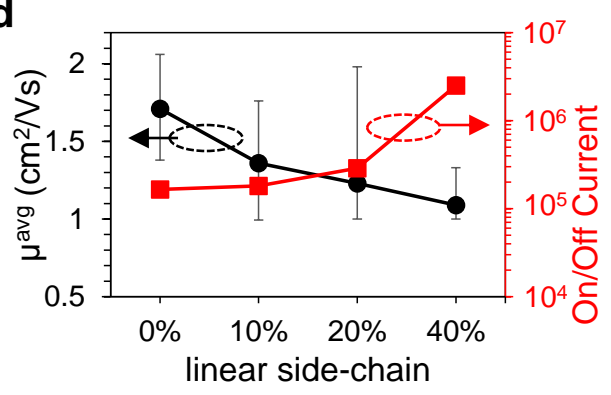

Figure 1. a) Chemical structure of DPP-based random co-polymer containing crosslinkable linear sidechains and linear, H-terminated PDMS crosslinker (top right); b) Schematic representation of the covalently crosslinked film. Arrows show charge transport direction; c) Measured molecular weight of the polymers by high-temperature size exclusion chromatography in $180{ }^{\circ} \mathrm{C} \mathrm{1,2,4-trichlorobenzene;} \mathrm{and} \mathrm{d)} \mathrm{Average} \mathrm{field-effect} \mathrm{mobility} \mathrm{and}$ on/off current of the non-crosslinked polymers. Values were summarized from more than 24 devices for each polymer. 


\section{WILEY-VCH}

a

\section{Hydrosilylation}
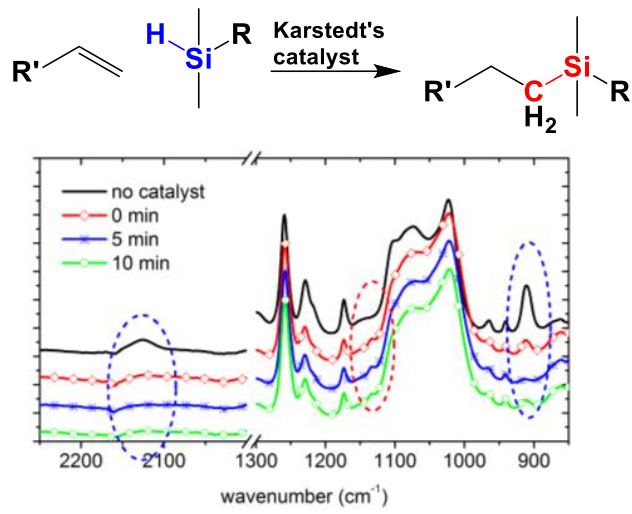

C

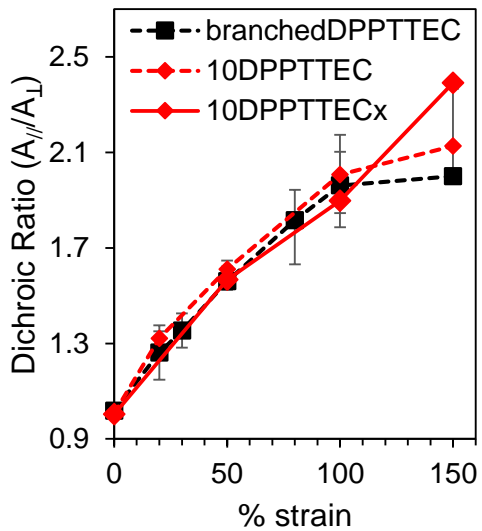

b
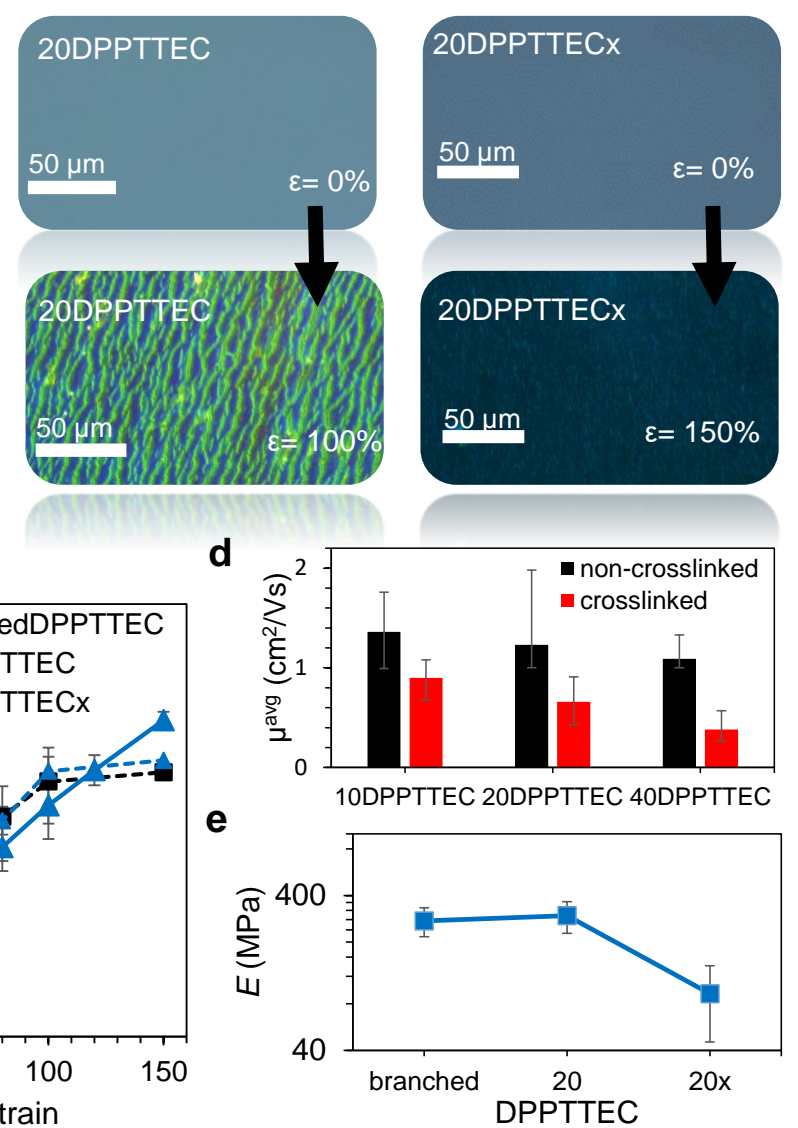

Figure 2. a) Reaction scheme (top) and attenuated total-reflectance Fourier transform infrared (ATR-FTIR) spectroscopy (bottom) of hydrosilylation crosslinking reaction; b) Optical microscope images of 20DPPTTEC (left) and 20DPPTTECx (right) in dark field mode. Microcracks are visible in 20DPPTTEC film at $\varepsilon=100 \%$, while no cracks are observable by optical microscopy for 20DPPTTECx at $\varepsilon=150 \%$; c) Dichroic ratio of 10DPPTTECx (left) and 20DPPTTECX (right) films with their non-crosslinked counterparts and control polymer branched-DPPTTEC over a range of strains; d) Average field-effect mobility of crosslinked polymers compared with non-crosslinked polymers. Values were summarized from more than 24 devices for each polymer; and e) Plot of the tensile modulus of branched-DPPTTEC, 20DPPTTEC and 20DPPTTECx. 


\section{WILEY-VCH}

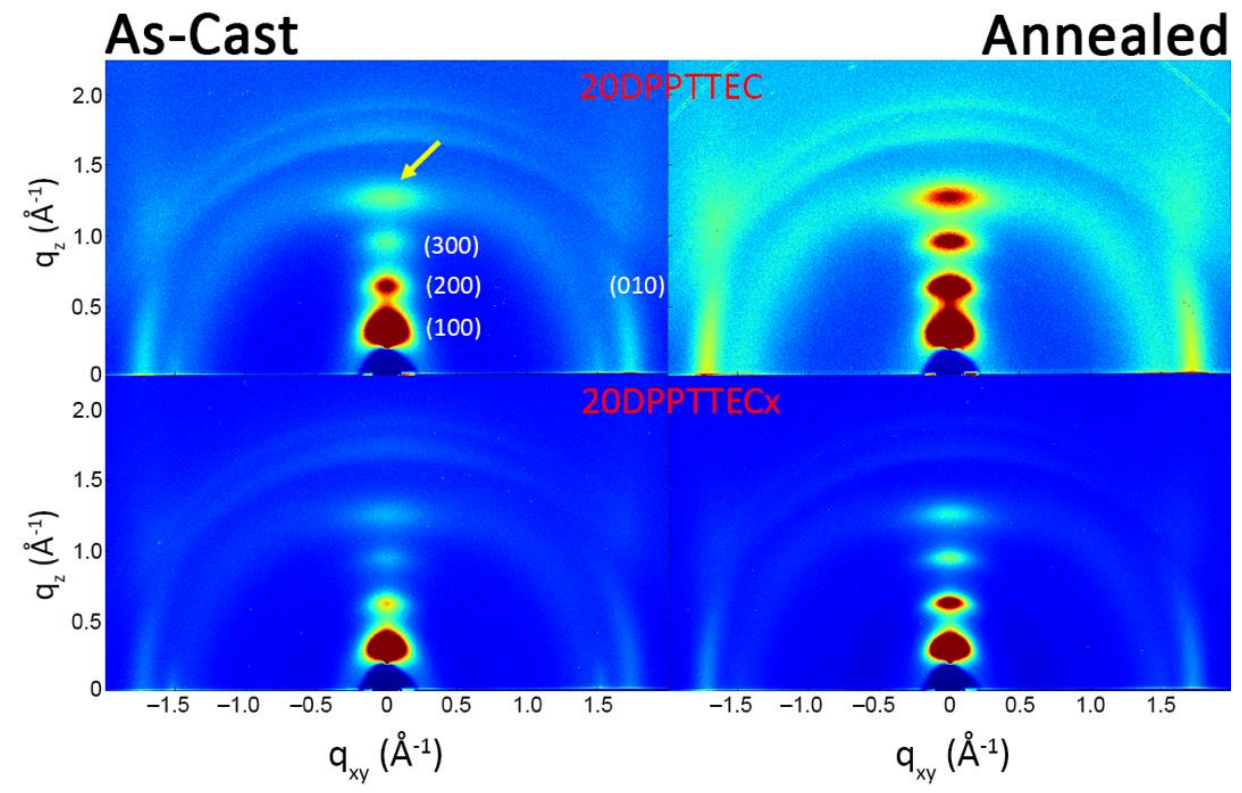

Figure 3. Grazing-incidence $X$-ray diffraction images of films spin-cast onto OTMS-treated Si substrates without (left) and with (right) thermal annealing at $160^{\circ} \mathrm{C}$ under vacuum. All images were scaled for exposure time and illuminated volume to provide a qualitative comparison across different samples. The films after annealing are more crystalline, and the intensity difference between the pristine and the crosslinked polymer suggests that the crosslinking decreases the thin film crystallinity. 


\section{WILEY-VCH}

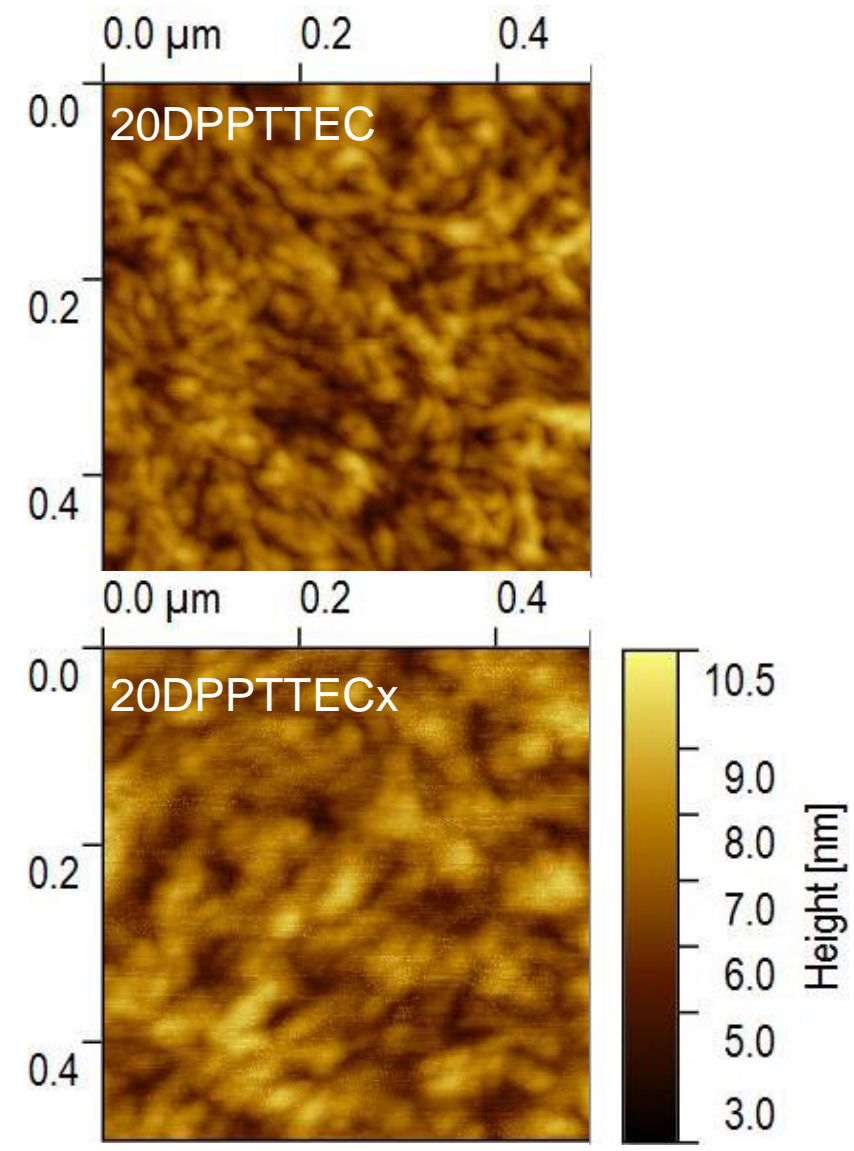

Figure 4. AFM tapping mode topographies of 20DPPTTEC and 20DPPTTECx films annealed at $160{ }^{\circ} \mathrm{C}$ under vacuum. An obvious nanofibrillar morphology was obtained for the neat film whereas the crosslinked film showed a decreased in nanofibrillar crystal domain. 


\section{WILEY-VCH}

a

Bottom Gate Top Contact (BGTC)

Au S/D Electrodes

Semiconductor

OTS-SiO ${ }_{2}$ Dielectric

$\mathrm{n}^{++} \mathrm{Si}$ Gate Electrode

Soft-Contact Lamination

Au S/D Electrodes OTS-SiO 2 Dielectric $\mathrm{n}^{++} \mathrm{Si}$ Gate Electrode

Semiconductor

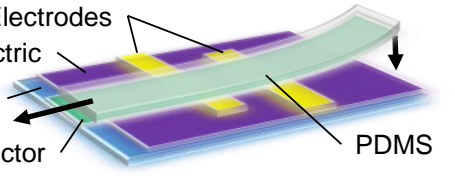

b

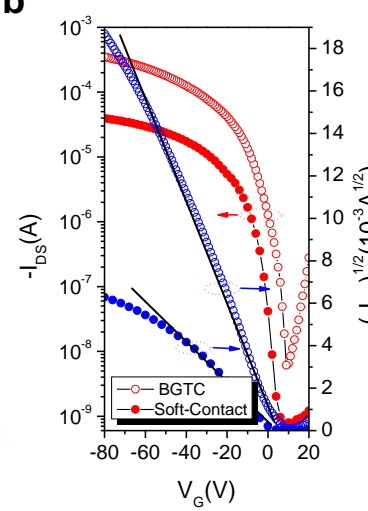

C

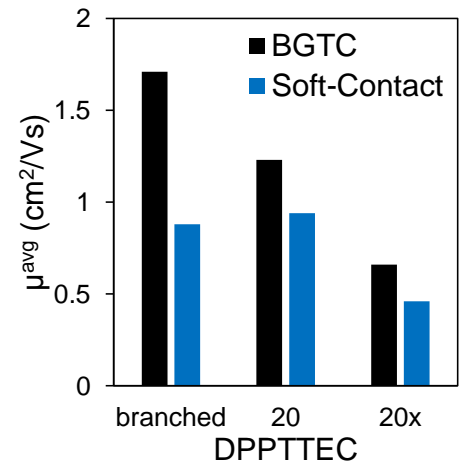

Figure 5. a) Schematic representation showing device structure of BGTC (top) and soft-contact lamination (bottom) OFET; b) Transfer characteristics of 20DPPTTECx as evaluated from BGTC and soft-contact lamination configuration $(L=50 \mu \mathrm{m}, W=1000 \mu \mathrm{m})$. The source-drain voltage was set to be $-100 \mathrm{~V}$ for all measurements; and c) Average field-effect mobility of branched-DPPTTEC, 20DPPTTEC, and 20DPPTTECx in the BGTC and soft-contact lamination configurations. 


\section{WILEY-VCH}

a
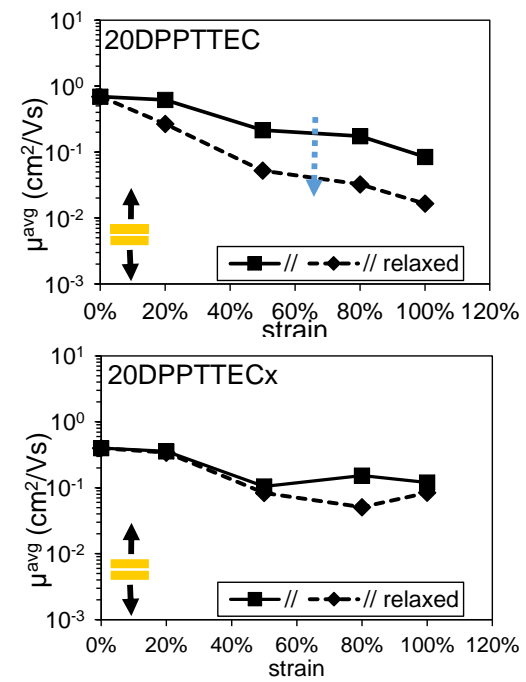

b
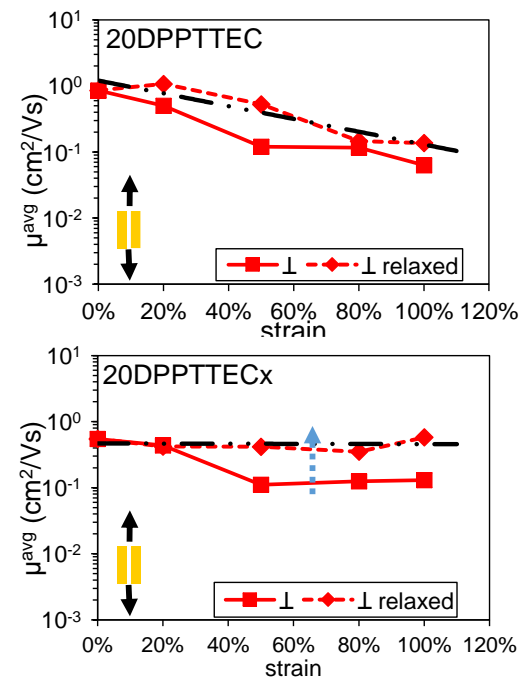

Figure 6. Average mobility evaluated from soft-contact lamination OFET devices $(L=50 \mu \mathrm{m}$, $W=1000 \mu \mathrm{m}$ ) of 20DPPTTEC and 20DPPTTECx upon strain and upon relaxation in the directions parallel $(/ /, a)$ and perpendicular $(\perp, b)$ to strain. 


\section{WILEY-VCH}

a
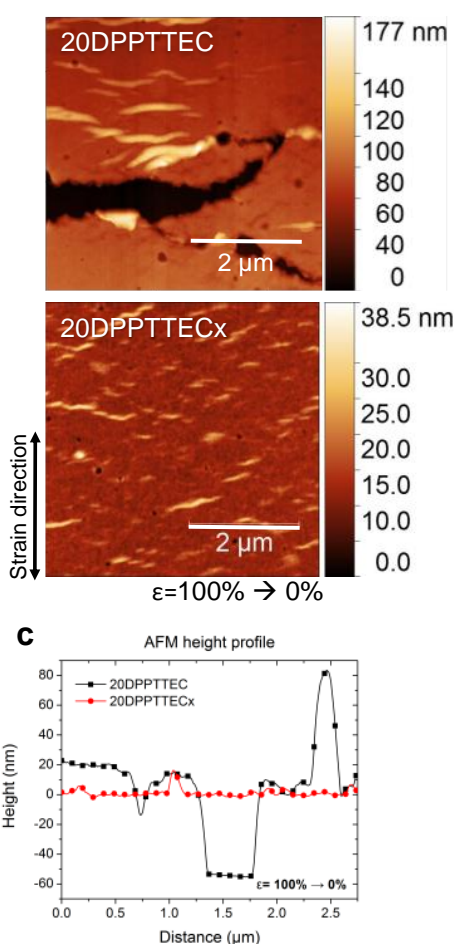

b
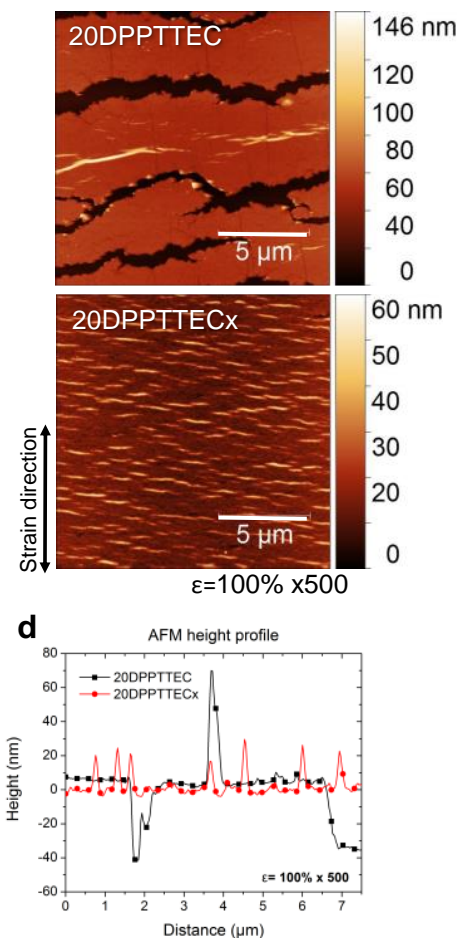

Figure 7. AFM images of 20DPPTTEC and 20DPPTTECx relaxed from a) 100\% strain and b) 500 cycles of $100 \%$ strain and their respective height profiles. The crosslinked polymer forms 20-nm-tall wrinkles upon strain and cyclic loading, whereas the pristine polymer forms microcracks and 60-nm-tall wrinkles. 


\section{WILEY-VCH}

a
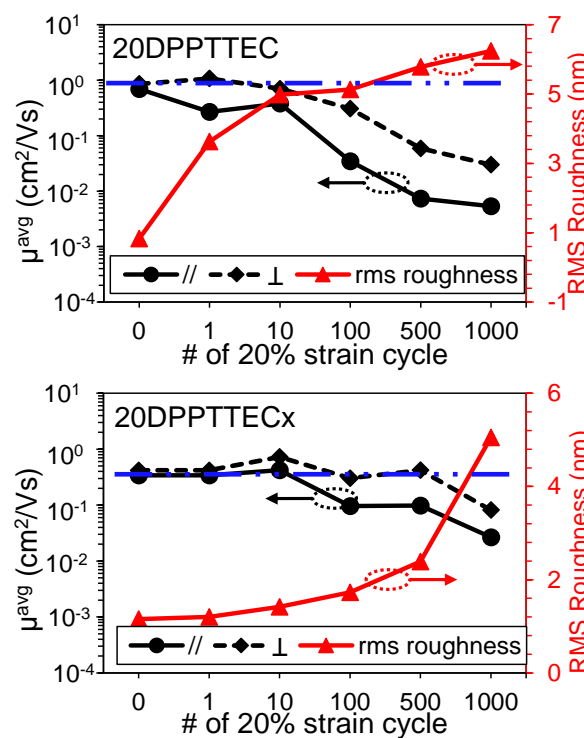

b
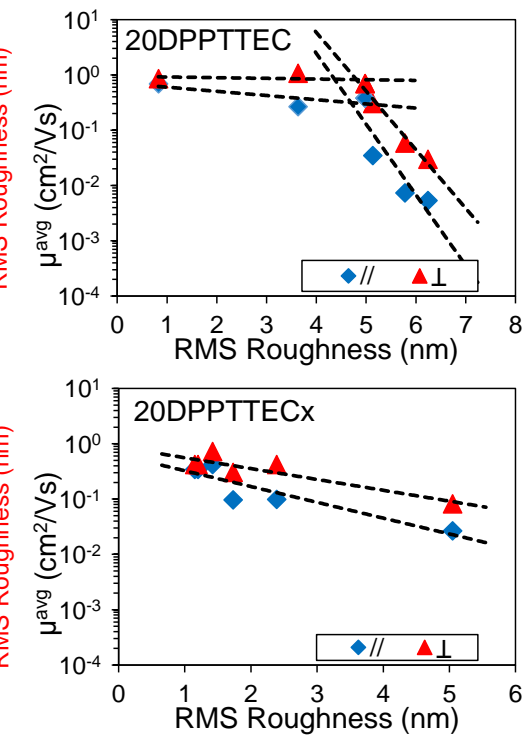

Figure 8. a) RMS roughness and average mobility of 20DPPTTEC (top) and 20DPPTTECx (bottom) with increasing cycles of $20 \%$ strain; b) Decay of average mobility with increasing RMS roughness in 20DPPTTEC (top) and 20DPPTTECx (bottom) films, with exponential fits to the data. 


\section{WILEY-VCH}

Improved elastic property in diketopyrrolopyrrole polymer is achieved by crosslinking with a flexible siloxane oligomer. An enhancement in fracture strain and yielding point; and a decrease in tensile modulus with film crystalinity is observed. The improved fatigue resistance is attributed to the covalent crosslinks that prevent irreversible sliding between polymer chains during cyclic loading.

Stretchable, semiconducting polymers, elastic, OFET

G. N. Wang, L. Shaw, Dr. J. Xu, Dr. T. Kurosawa, Dr. B. C. Schroeder, Dr. J. Y. Oh, Dr. S. J. Benight, Prof. Z. Bao*

Inducing elasticity through oligo-siloxane crosslinks for intrinsically stretchable semiconducting polymers

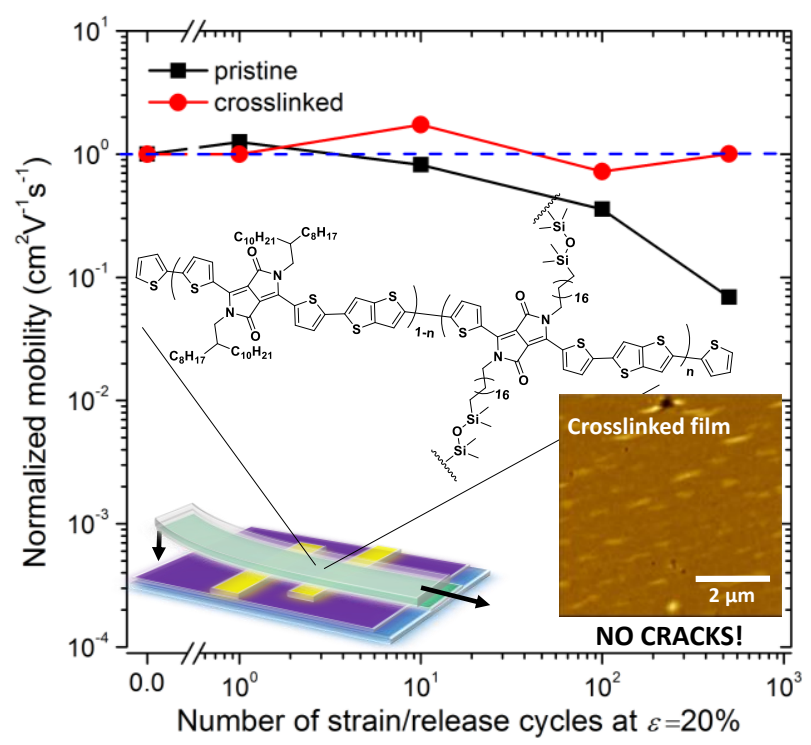

Copyright WILEY-VCH Verlag GmbH \& Co. KGaA, 69469 Weinheim, Germany, 2013. 